\title{
Abstracts
}

\section{Informal Care and Social Support}

\section{John Bond}

Aluma Kopito Motenko, The frustrations, gratifications and wellbeing of dementia caregivers. The Gerontologist, 29 ( (1989), I66-72.

Dementia is an extremely incapacitating disease. It affects the sufferer in many and unpredictable ways. A gentle person may become aggressive and harmful to their spouse or become confused. The literature on the burden on informal carers has been extensively documented in Europe and North America. A growing interest in the literature is the rewards of caring to the informal carer. Love, affection, reciprocity and commitment have been cited as primary reasons for providing care. Caring allows informal carers to express these basic human emotions. Motenko sets out to explore the relationship between carer well-being and the frustrations and gratifications of caring for a significant other.

Motenko tests two hypotheses:

I. Caregivers who experience greater gratification from caring will have higher general well-being than caregivers who experience less gratification.

2. Caregivers who experience greater frustration from caring will have lower general well-being than caregivers who experience less frustration.

In the course of the paper a number of related hypotheses are also tested. The study used to test these hypothesis is comprehensively reported. In summary 50 women caring for a spouse with dementia living in Massachusetts were studied. Psychometrically-valid measures of the following concepts were used:

- general well-being - perceived anxiety, perceived depression, perceived self-control, perceived health and perceived vitality;

- gratification - with emotional support, marriage and having husband at home;

- frustration - frustration with activities of daily living, financial strain and with time for self, constrained by caring, frustration with household chores and with change in emotional support;

- the mean of caring - reciprocity, responsibility and providing tender loving care. 
The analysis of interviews suggested that frustration and gratification from caring are strongly associated with the importance of continuity in the closeness of the marital relationship, in the meaning of caring, in the social support network and in patient characteristics. The stated hypotheses have not been rejected by this analysis. From the analyses Motenko suggests the following:

I. Spouses who have enjoyed long, meaningful and enduring relationships with their husbands before the illness benefit from sustaining their caring role despite the great burdens endured.

2. Spouses who do not derive gratification from caring are those who are unable to maintain continuity in their relationship with their husbands.

3. A caregiving relationship characterized by responsibility connotes a break in the marital relationship and can contribute to the burdens rather than the gratifications of caregiving.

4. Caregivers who perceive continued companionship, affection and continuity in life patterns from their marriages will derive greater gratification from caregiving than those who perceive discontinuity in social support.

5. The longer the husbands had been sufferers the less frustrated caregivers were with changes in emotional support.

This paper is an important contribution to the literature because it challenges a number of current assumptions held by policy makers and professionals alike. This study highlights the relative unimportance of duration of the carer role or severity of the sufferer's symptoms in predicting the burden of informal care. It also highlights the greater reactivity of caregivers to the context of care than generally assumed. The meaning that individuals give to their own carer role and their perceptions of why they care are crucial.

The conventional assumption that the provision of intensive care by a spouse of the kind required for the support of a dementia sufferer erodes the caregivers personal and psychological resources is challenged by these analyses. The majority of caregivers adapted well to their new responsibilities and to the progressive emotional withdrawal of their demented husbands.

The conventional wisdom that institutionalisation of people with dementia is required to. preserve the physical and mental health of elderly caregivers is also challenged. Long, meaningful marriages that continue to remain meaningful despite the existence of dementing illness have been shown to contribute to caregivers gratification and well-being.

A conclusion to be drawn from this paper is that practitioners should 
promote caregivers well-being by preserving continuity and minimising disruption in the lives of caregivers. A goal for social policy research should be to identify how the gratifications and frustrations of caring influence the risk of stress and ill health among all types of caregivers.

Mary Gilhooly and Janice Whittick, Expressed emotion in caregivers of dementing elderly. British Fournal of Medical Psychology, 62 (1989), $265-72$.

One way of measuring the level of frustration among caregivers would be to measure expressed emotion (EE). Gilhooly and Whittick describe an attempt to measure EE by counting the number of critical comments caregivers of dementia sufferers made about their elderly relative with dementia. The study focused on 24 co-resident and 24 non-co-resident caregivers of elderly people with dementia attending two Scottish day hospitals. Data were collected through semi-structured interviews with caregivers and expressed emotion was measured by counting the number of critical comments made about the elderly relative with dementia during the interview and during the first hour of the interview. (As one would expect the number of negative comments was correlated with the length of interview). An example of a negative comment is:

I feel like telling him to go to hell and not going back... he's such an old sod... I often feel like it, very often.

Using the number of expressed emotions as the dependent variable the authors explore its relationship with a number of independent variables including: demographic characteristics of subject and carer; subject's and carer's level of mental and physical impairment; carers contact and satisfaction with other relatives; social support assessment; help from statutory services; carer's perception of the quality of relationship with subject and an index assessing preference for institutional care.

The number of critical comments ranged from none to 78 (mean $=$ I 2.67 ; S.D. $=17.48$ ). Compared to studies of people with other psychiatric disorders the number of critical comments was quite high, but as Gilhooly and Whittick point out this may be a reflection of interviewing procedures including length of interview. There was a significant association between EE and carer's psychological wellbeing. Men made fewer critical comments than women and those with the least contact with friends made more critical comments about 


\section{John Bond}

subjects. Perhaps not surprisingly the quality of the relationship prior to the onset of dementia was strongly associated with EE.

The authors conclude that the assessment of $\mathrm{EE}$ could be an important predictor of breakdown in informal care.

Betty Risteen Hasselkus, Meaning in family caregiving: perspectives on caregiver/professional relationships. The Gerontologist, 28 ( 1988 ), 686-91.

The focus of this paper is on the meaning of caregiving. Much of research on caregiving assumes an instrumental approach to caregiving. In addition to the tasks that carers perform they are also involved in decision making, protecting the subjects' self-images and protection from and awareness of being cared for. The study reported in this paper was undertaken to understand the meaning of the caregiving experience and the patterns of informal learning embedded in that meaning. Meaning was defined as those values, beliefs and principles that people use to organise their behaviour and to interpret their experience.

Ethnographic methods were used comprising four separate interviews with i 5 family carers. The structure of subsequent interviews was dictated by the data collected in the first interview with carers. From the interviews a number of themes of meaning for carers emerged:

- a concern for self, a sense of personal capabilities and a heightened sense of personal causation comprised carers' sense of self;

- a pervasive theme of managing occurred throughout the interview data. It was often maintained by the use of elaborate rituals and routines;

- a sense of doom about the future pervaded the interviews and was often related to a worsening in the subject's condition and the carer's perception about their continuing ability to cope;

- a sense of fear of change which was related to their sense of the future and a sense of risk which related to their concern for the safety of the subject was common theme in interviews;

- changes in role and responsibility were perceived by some carer respondents;

- the caregiving role was. full of tensions between the carer and subject and between other providers of care. A major tension was the frequent expectation held by the subject, that the carer should be available at all times;

- the relationship between formal and informal carers was not always ideal. Tensions existed between professionals and family members in 
a number of domains. The data highlight tensions in relation to the ownership of expert knowledge and ways in which carers reinterpret instructions given by professionals.

Thus this study complements previous research which highlighted the invisible work of carers and carers' questioning of the knowledge and advice of professionals. The different themes emerging form these data highlight the reflective practice of the carers. If recognised by professionals who modify their input to share ownership of interaction with the carer then collaboration in caring can proceed more smoothly. It is necessary for carers and professionals to recognise the other's context of meanings and to make meanings accessible to each other. Thus a professional's approach to informal carers should be one which emphasises the professional's willingness to take the perspective of the carer.

Emily K. Abel, the ambiguities of social support: adult daughters caring for frail elderly parents. Journal of Aging Studies, 3 ( I989), 2 I I-3o.

This article explores the extent to which family caregivers can rely on members of their social networks for help. Previous research suggests that help from members of carers social networks helps shield carers from stress. Abel argues that these studies have failed to provide an adequate conceptualisation of support. The objective of this article is to rectify this deficiency of the literature. Abel suggests that social support can be conceptualised as having the following components: instrumental assistance, emotional sustenance, affirmation and companionship.

The study described examines the perceptions of adult daughters caring for frail elderly parents of the social support they received. It focuses on their perceptions of the type of support they received from various sources, as well as the barriers they perceived to securing additional support. A sample of 40 caucasian women were contacted using snowballing techniques. Semi-structured open-ended interviews were undertaken and analysed using qualitative methods.

Most women were caring for their mothers, seven for their fathers and six for both parents. Over half were caring for parents with a diagnosed dementia. In describing their caring roles virtually all respondents claimed that it was a source of strain but a number found caring rewarding and fulfilling. Few anticipated caring for their parents, had little warning or felt prepared to take on the role. The 


\section{John Bond}

opportunity costs of caring were substantial. In relation to paid employment, some women forfeited promotion, others took on less demanding jobs or worked less hours while others gave up paid work altogether. Few were able to protect their family lives and leisure time. Most emphasised the emotional dynamics of caring for an elderly parent. In short Abel found 'parent care involves constant tensions between attachment and loss, pleasing and caring, seeking to preserve an older person's dignity and exerting unaccustomed authority, overcoming resistance to care and fulfilling extravagant demands, reviving a relationship and transforming it' (p. 216).

The respondents wanted others in their social network to value their caring role and to provide additional advice and support. They indicated that members of their social network could exacerbate distress as well as alleviate it. Respondents defined social support in a variety of ways and noted a number factors which inhibited them from seeking greater help from their family and friends. In particular, concern for the well-being of significant others compelled the caregivers to submerge their own needs. However, despite this tendency the women did receive support from significant others in the form of advice about decisions, information that permitted them to plan ahead and assistance with household chores. Respondents were less forthcoming about how the extent to which social relationships enabled them to deal more effectively with the interpersonal and intra-psychic demands.

Abel concludes by highlighting two related issues for future research about social support. First, by focussing on the different components of social support past research has missed the essential meaning of social support to the actors involved. In the future it may be more useful to focus on social support as a whole. Related to this has been the tendency to focus on the individual as the unit of analysis. Future research needs to consider the caring unit as a whole and to understand the interrelationships which exist within the organisation of informal care.

\section{Comment}

In an editorial in a recent edition of The Gerontologist Zarit posed the question: 'Do we need another stress and caregiving study? '1 Certainly the literature looking at the social costs of caring has mushroomed in the last decade in Europe and North America. However, much of the literature is derived from small-scale studies which have not been subject to rigorous qualitative analysis. In methodological terms there is still a need to undertake a number of large-scale studies to test the 
numerous hypothesis about the relationship between the caring role and personal well-being. The papers reviewed in this issue also suggest that the relationship between the caring role and carer well being is exceptionally complicated and further rigorous qualitative and quantitative studies will be required to unravel the differences which exist between different cultures and different welfare systems. As well as undertaking further, perhaps, more sophisticated non-descriptive analysis it would be helpful to undertake a meta-analysis of some of these studies in order to sift the effects of poorly constructed studies, small sample sizes, national and cultural differences and the changing nature of the caring task. Future research must be coordinated and focus on new hypotheses rather than simply replicate the best of what has already been achieved.

\section{NOTE}

1 Zarit, S. H., Do we need another 'stress and caregiving' study? The Gerontologist, 29 ( I 989 ), I $47-8$.

Health Care Research Unit, University of Newcastle upon Tyne

\section{Morbidity and Mortality Trends}

\section{Tony Warnes}

J. F. Fries, The compression of morbidity: near or far. Milbank Quarterly, 67, 2 (1989), 208-32.

It is ten years since the initial presentation of the compression of morbidity thesis (Fries, 1980). In this article, the author restates and refines his arguments and reviews the evidence from a number of large scale population and clinical trials. The original predictions were that life-expectancy gains at advanced ages would slow (in the United States population), that the male:female life-expectancy gap would narrow, and that reductions in age-specific incidence rates for chronic illness would exceed reductions in age-specific mortality rates. This last underpinned the derivative prediction that the variability in ages of death would decrease. Higher fractions of the population would survive until near the modal age of death, following which the survival curve would descend more steeply than at present: the survival curve would become more 'rectangular'. The projected reduction of the duration of chronic illness also implied a per capita reduction in health-care costs. It 